\title{
TESTE DE CONDUTIVIDADE ELÉTRICA PARA AVALIAÇÃO DA QUALIDADE FISIOLÓGICA DE SEMENTES DE AZEVÉM (Lolium multiflorum L.) ${ }^{1}$
}

\author{
RODRIGO RAMOS LOPES²; LÚCIA BRANDÃO FRANKE ${ }^{3}$
}

\begin{abstract}
RESUMO - O teste de condutividade elétrica tem potencial para ser empregado no controle de qualidade pelas empresas produtoras de sementes, no entanto há fatores que podem afetar os resultados obtidos. O objetivo do trabalho foi avaliar o efeito do número de sementes, volume de água e o tempo de embebição na condutividade elétrica da solução, para avaliação do vigor em sementes de azevém. $\mathrm{O}$ experimento foi conduzido utilizando-se quatro lotes de sementes de azevém. $\mathrm{O}$ teste foi realizado à temperatura de $25^{\circ} \mathrm{C}$, por períodos de $1,2,4,6,8$ e 24 horas de embebição, em 50 e $75 \mathrm{~mL}$ de água deionizada, utilizando-se 50 e 100 sementes. Os tratamentos constituíram um fatorial, utilizando-se o delineamento inteiramente casualizado, com quatro repetições. A utilização de quatro subamostras de 50 sementes puras embebidas em $50 \mathrm{~mL}$ de água deionizada é promissora para a realização do teste de condutividade elétrica em sementes de azevém a partir de uma hora de embebição das sementes.
\end{abstract}

Termos para indexação: potencial fisiológico, integridade de membranas, vigor.

\section{ELECTRICAL CONDUCTIVITY TEST TO EVALUATE THE PHYSIOLOGICAL QUALITY OF RYEGRASS (Lolium multiflorum L.) SEEDS}

\begin{abstract}
The electrical conductivity test has the potential to be used by seed companies in quality control but some factors can affect the results of the EC. The object of this study was to evaluate the effect of the number of seeds, water volume and the imbibition time on the electrical conductivity of the solution, on ryegrass seed vigour. Four lots of common ryegrass seeds were used. The test was made at $25^{\circ} \mathrm{C}$, at imbibition testing times of $1,2,4,6,8$, and 24 hours, in 50 and $75 \mathrm{~mL}$ water, using 50 and 100 seeds. The treatments constituted a factorial, using a completely randomized design with four repetitions. The use of four subsamples of 50 pure seeds soaked in $50 \mathrm{~mL}$ of deionized water is promising for the use of the electrical conductivity test of ryegrass seeds conducted after 1 hour of soaking.
\end{abstract}

Index terms: physiological potential, integrity of membranes, vigour.

\section{INTRODUÇÃO}

O azevém anual (Lolium multiflorum L.) é a forrageira de mais ampla utilização no Rio Grande do Sul (TerraLopes et al., 2009), assim como na maior parte das regiões temperadas e subtropicais do mundo, destaca-se entre as espécies mais difundidas mundialmente (Bressolin, 2007). Apresenta resistência ao frio, qualidade nutricional e potencial de produção de matéria seca (Pereira et al., 2008). Possui considerável capacidade de rebrote e boa
${ }^{1}$ Submetido em 07/11/2008. Aceito para publicação em 04/06/2009.

${ }^{2}$ Eng. Agr., Dr., Programa de Pós-graduação em Zootecnia, Faculdade de Agronomia, Universidade Federal do Rio Grande Sul, Bolsista CNPq, e-mail: lopezhsf@hotmail.com
${ }^{3}$ Eng. Agr., Dr., Prof. do Dept. de Plantas Forrageiras e Agrometeorologia da Faculdade de Agronomia/UFRGS, Cx. Postal 15100, 91501-970, Porto Alegre /RS, e-mail: lbfranke@ufrgs.br 
ressemeadura natural, sendo bem aceito pelos animais podendo produzir de duas a seis toneladas de matéria seca por hectare (Santos et al., 2002). Estas características justificam a preferência dessa forrageira, pelos produtores na formação de pastagens de clima temperado, tanto em cultivo puro como consorciado.

O teste de germinação é o procedimento oficial para avaliar a capacidade das sementes produzirem plântulas normais em condições ideais, mas nem sempre revela diferenças de desempenho entre lotes de sementes durante o armazenamento ou em campo (Carvalho e Nakagawa, 2000). Desta maneira, é importante avaliar o vigor das sementes como complemento às informações fornecidas pelo teste de germinação. Para isso, vários procedimentos têm sido usados na avaliação do vigor em sementes de azevém, dentre eles o teste de envelhecimento precoce (Garcia e Menezes, 1999) e o teste de envelhecimento acelerado com uso de solução salina, no entanto, são testes de relativa demora na obtenção dos resultados.

Nas últimas décadas, uma das principais exigências das empresas de sementes tem sido relacionada à avaliação da qualidade das sementes de forma rápida e eficiente, de modo a permitir a agilização das tomadas de decisões, principalmente no que se refere às operações de colheita, processamento e comercialização. Segundo Hampton e Coolbear (1990), em função das limitações do tempo requerido para o teste de germinação, têm sido contínuo o interesse pelo potencial das propriedades fisiológicas e bioquímicas das sementes como índices de vigor. Os testes rápidos de vigor mais estudados estão relacionados com eventos iniciais da seqüência de deterioração das sementes tais como, degradação das membranas celulares e a redução das atividades respiratórias e biossintéticas (Dias e Marcos Filho, 1996).

A condutividade elétrica tem mostrado boa relação com a emergência das plântulas em campo e separação de lotes em diferentes níveis de qualidade (Vieira e Krzyzanowski, 1999), tanto que estes motivos têm sido a maior razão para o seu emprego em pesquisa. O teste é baseado na menor velocidade de estruturação das membranas por sementes menos vigorosas, quando embebidas em água, tendo como conseqüência maior liberação de exsudatos para o exterior da célula e, portanto, maior condutividade elétrica que aquelas mais vigorosas (Marcos Filho, 2005). Assim, considera-se o vigor das sementes inversamente proporcional à leitura da condutividade elétrica (Vieira e Krzyzanowski, 1999).

Desta forma, o presente trabalho teve como objetivo avaliar a eficiência do teste de condutividade elétrica na determinação do vigor de sementes de azevém anual, procurando-se obter informações rápidas que possam ser utilizadas pela indústria de sementes.

\section{MATERIAL E MÉTODOS}

O experimento foi conduzido no Laboratório de Análise de Sementes, no Departamento de Plantas Forrageiras e Agrometeorologia, da Faculdade de Agronomia da Universidade Federal do Rio Grande do Sul. Utilizaram-se quatro lotes de azevém (Lolium multiflorum L.) de qualidade fisiológica diferente (L1, L2, L3 e L4).

O grau de umidade de cada amostra foi determinado em duas repetições de $5 \mathrm{~g}$ para todos os lotes, utilizando-se estufa a $105{ }^{\circ} \mathrm{C} \pm 3{ }^{\circ} \mathrm{C}$ durante 24 horas, sendo calculada por diferença de massa, com base na massa úmida das sementes, conforme as Regras para Análise de Sementes (Brasil, 2009).

$O$ teste de germinação foi realizado utilizando-se 400 sementes por lote, usando quatro repetições de 100 sementes, em germinador, a temperatura constante de $20{ }^{\circ} \mathrm{C}$, por 14 dias (Brasil, 2009). Os resultados foram expressos em porcentagem de plântulas normais obtidas no décimo quarto dia (Brasil, 2009); primeira contagem de germinação - realizada durante a realização do teste de germinação, considerando-se a porcentagem de plântulas normais obtidas no quinto dia após a semeadura.

A emergência das plântulas foi realizada em casa de vegetação, com 400 sementes por lote, com quatro subamostras de 100, semeadas em bandejas plásticas contendo substrato comercial (solo orgânico) com quantidade de água equivalente a $70 \%$ da capacidade de campo. A contagem foi feita após um período de 14 dias, computando-se a porcentagem de plântulas emergidas para cada lote.

O teste de envelhecimento acelerado - adotou-se a metodologia recomendada por Garcia e Menezes (1999). Uma única camada de sementes foi colocada sobre tela metálica acoplada à caixa plástica gerbox, contendo $40 \mathrm{~mL}$ de água ao fundo. As caixas foram tampadas, de modo a obter $100 \%$ UR em seu interior, sendo mantidas em incubadora BOD a $41{ }^{\circ} \mathrm{C}$, durante 48 horas. Decorrido o período de exposição $\left(41{ }^{\circ} \mathrm{C} / 48 \mathrm{~h}\right)$, quatro sub-amostras de 100 sementes foram colocadas para germinar, seguindo método descrito para o teste de germinação. Uma única avaliação foi realizada no $14^{\circ}$ dia após a semeadura, computando-se a porcentagem de plântulas normais.

Envelhecimento acelerado com solução salina 11\% utilizou-se a metodologia descrita para o método tradicional, 
porém, substituindo-se a água no interior das caixas gerbox por $40 \mathrm{~mL}$ de solução não saturada de $\mathrm{NaCl}(11 \mathrm{~g}$ de $\mathrm{NaCl}$ em $100 \mathrm{~mL}$ de água), estabelecendo um ambiente com umidade relativa de $94 \%$.

Teste de condutividade elétrica - foram estudadas variações no volume de água deionizada $(50$ e $75 \mathrm{~mL})$, período de embebição (1, 2, 4, 6, 8 e 24 horas) e número de sementes utilizadas (50 e 100). Foram avaliadas quatro repetições de 50 e 100 sementes, fisicamente puras, pesadas com precisão de duas casas decimais $(0,01 \mathrm{~g})$, colocadas para embeber em copos plásticos $(200 \mathrm{~mL})$ e mantidas em câmara tipo $\operatorname{BOD}\left(25^{\circ} \mathrm{C}\right)$ durante o período de embebição. Após cada período de embebição, a condutividade elétrica da solução foi determinada por meio de leituras em condutivímetro DIGIMED modelo DM 3, com os resultados expressos em $\mu \mathrm{S} . \mathrm{cm}^{-1} \cdot \mathrm{g}^{-1}$. À exceção dos fatores estudados, o teste foi conduzido conforme descrito por Vieira e Krzyzanowski (1999).

O delineamento experimental utilizado foi o inteiramente casualizado, com quatro repetições. Os dados obtidos no teste de condutividade elétrica foram analisados em esquema fatorial $4 \times 2 \times 2 \times 6$ ( 4 lotes, 2 números de semente, 2 volumes de água e 6 períodos de embebição) através do procedimento PROC GLM do programa Statistical Analysis System ${ }^{\circledR}$ versão 9.1.3 (SAS, 2004) e, em caso de diferença significativa, as médias foram comparadas pelo teste de Tukey a $5 \%$ de probabilidade. Os dados obtidos em porcentagem foram submetidos a testes de normalidade que indicaram a não necessidade de transformação.

\section{RESULTADOS E DISCUSSÃO}

Na Tabela 1, pode-se observar o teor de água inicial dos lotes avaliados e o teor de água atingido após a realização do teste de envelhecimento acelerado tradicional e envelhecimento acelerado modificado (com o uso de solução a $11 \%$ de $\mathrm{NaCl}$ ). Os valores foram semelhantes para os quatro lotes, com variação de até 0,1 (umidade inicial), 0,52 (envelhecimento acelerado) e 0,71 pontos porcentuais (envelhecimento acelerado modificado), todas inferiores à amplitude máxima aceita que é de 1 a 2 pontos percentuais (Marcos Filho, 1999). Segundo o mesmo autor, diferenças acentuadas podem provocar alterações na velocidade de umedecimento das sementes durante o tratamento de envelhecimento e determinar diferenças na intensidade de deterioração. O teor de água das sementes é de extrema importância para padronização de um método, bem como na obtenção de resultados uniformes entre laboratórios e dentro de um mesmo laboratório, por ocasião da realização de um teste de condutividade elétrica.

TABELA 1. Teor de água antes e após os testes de envelhecimento acelerado (EA) e envelhecimento acelerado modificado (EA $11 \% \mathrm{NaCl}$ ) em quatro lotes de sementes de Lolium multiflorum $\mathrm{L}$. com diferentes níveis de qualidade fisiológica.

\begin{tabular}{cccc}
\hline \multirow{2}{*}{ Lotes } & Inicial & $\begin{array}{c}\text { EA } \\
\left(41^{\circ} \mathrm{C} .48 \mathrm{~h}\right)\end{array}$ & $\begin{array}{c}\text { EA } 11 \% \mathrm{NaCl} \\
\left(41^{\circ} \mathrm{C} .24 \mathrm{~h}\right)\end{array}$ \\
\cline { 2 - 4 } & \multicolumn{3}{c}{ Teor de água $(\%)$} \\
\hline L1 & $12,48 \mathrm{a}$ & $25,90 \mathrm{a}$ & $12,79 \mathrm{a}$ \\
L2 & $12,47 \mathrm{a}$ & $25,38 \mathrm{a}$ & $12,91 \mathrm{a}$ \\
L3 & $12,49 \mathrm{a}$ & $25,39 \mathrm{a}$ & $12,20 \mathrm{a}$ \\
L4 & $12,57 \mathrm{a}$ & $25,43 \mathrm{a}$ & $12,20 \mathrm{a}$ \\
\hline C.V. & 1,1 & 0,9 & 2,7 \\
\hline
\end{tabular}

Os valores seguidos pela mesma letra, na coluna, não diferem entre si pelo teste de Tukey $(\mathrm{P} \leq 0,05)$.

Pelos resultados observados na Tabela 2, verifica-se que o lote 1 foi o mais vigoroso, pois melhores resultados para germinação e vigor, pelos testes de primeira contagem, envelhecimento acelerado, envelhecimento acelerado modificado e emergência de plântulas em solo foram obtidos. Pelos testes de vigor, o lote 4 foi inferior na primeira contagem, envelhecimento acelerado, envelhecimento acelerado modificado e emergência de plântulas em solo, enquanto os lotes 2 e 3 apresentaram-se semelhantes estatisticamente em todos os testes anteriormente citados. Os testes de vigor foram mais sensíveis ao avaliar a qualidade das sementes dos diferentes lotes estudados. Isso porque eles permitiram inferir numa avaliação mais nítida da qualidade fisiológica, fornecendo maiores subsídios para a separação desses lotes em diferentes níveis de vigor, quando se comparou os resultados destes com os de germinação.

Para ser avaliado como eficiente, um teste de vigor deve proporcionar uma classificação dos lotes em diferentes níveis de vigor, de maneira proporcional à da emergência das plântulas no campo (Marcos Filho, 1999) e não somente apresentar alta correlação com o mesmo. Neste experimento houve a necessidade de uma adaptação, efetuando-se um teste de emergência em solo em condições ambientais (sem controle de temperatura e luz). Entretanto, todos os testes de laboratório apresentaram correlações positivas com a porcentagem de emergência em solo (Tabela 3). Destacou- 
se pelos altos graus de associação com a emergência, o teste de primeira contagem $(\mathrm{r}=0,99)$ e o teste de envelhecimento acelerado $(\mathrm{r}=0,96)$. A primeira contagem da germinação é utilizada por alguns pesquisadores como índice de vigor pela facilidade de sua execução e quando há grande diferença no vigor dos lotes, oferecendo um indicativo aproximado do potencial fisiológico das sementes (Popinigis, 1985). Da mesma maneira, o teste de envelhecimento acelerado está diretamente relacionado ao potencial de conservação das sementes e, por esse motivo, é considerado um dos mais sensíveis para a avaliação do vigor, entre os disponíveis (Marcos Filho, 1999).

TABELA 2. Porcentagem de germinação, primeira contagem de germinação, envelhecimento acelerado (EA), envelhecimento acelerado modificado (EA $11 \%$ NaCl) e emergência (EMG) em quatro lotes de sementes de Lolium multiflorum L. com diferentes níveis de qualidade fisiológica.

\begin{tabular}{cccccc}
\hline \multirow{2}{*}{ Lotes } & Germinação & $1^{\mathrm{a}}$ Contagem & EA & EA 11\% NaCl & EMG \\
\cline { 2 - 6 } & & & Médias $(\%)$ & & \\
\hline L1 & $83 \mathrm{a}$ & $77 \mathrm{a}$ & $75 \mathrm{a}$ & $81 \mathrm{a}$ & a \\
L2 & $63 \mathrm{~b}$ & $39 \mathrm{~b}$ & $50 \mathrm{~b}$ & $59 \mathrm{~b}$ & $46 \mathrm{~b}$ \\
L3 & $52 \mathrm{c}$ & $38 \mathrm{~b}$ & $43 \mathrm{~b}$ & $48 \mathrm{bc}$ & $42 \mathrm{~b}$ \\
L4 & $43 \mathrm{~d}$ & $34 \mathrm{c}$ & $41 \mathrm{~b}$ & $39 \mathrm{c}$ & $39 \mathrm{c}$ \\
\hline C.V & 2,4 & 2,0 & 8,8 & 6,6 & 5,7 \\
\hline
\end{tabular}

Os valores seguidos pela mesma letra, na coluna, não diferem entre si pelo teste de Tukey $(\mathrm{P} \leq 0,05)$.

TABELA 3. Coeficientes de correlação linear simples (r) entre os testes de laboratório e a emergências (EMG) em sementes de quatro lotes de sementes de Lolium multiflorum L. com diferentes níveis de qualidade fisiológica.

$$
\text { Correlações }
$$

Teste de germinação $\times$ Emergência

Primeira contagem de germinação $\times$ EMG

Envelhecimento acelerado $\times$ EMG

Envelhecimento acelerado modificado $\times$ EMG

** Significativo pelo teste $\mathrm{t}$, a $1 \%$.

Os resultados do teste de condutividade elétrica, envolvendo as combinações de número de sementes e volumes de água nos diferentes períodos de embebição, são apresentados nas Tabelas 4, 5, 6 e 7. Por meio da análise da variância, verificaram-se efeitos significativos dos fatores estudados. Também foi observada interação significativa entre os lotes e período de embebição e número de sementes e período de embebição. As leituras da condutividade elétrica permitiram verificar, de maneira geral, que as diversas combinações de número de sementes (50 e 100) e volume de água $(50$ e $75 \mathrm{~mL})$ apresentaram semelhança na distinção dos lotes em relação ao seu potencial fisiológico. Ocorreram algumas variações na classificação dos lotes quando se comparou os dados de condutividade elétrica com a germinação e os testes de vigor utilizados para a caracterização do perfil dos lotes. Porém, todos os testes destacaram o lote 1 como sendo o de melhor qualidade com o sistema de membranas celulares mais íntegro ou com maior capacidade de restabelecer sua integridade, durante a embebição, menor lixiviação de eletrólitos, indicando maior vigor, como sugerem os estudos em outras culturas (Vieira e Krzyzanowski, 1999; Vieira et al., 2002; Dias et al., 2006), confirmando os resultados observados no teste de emergência em solo.

A redução do volume de água, quando se mantiveram constantes os outros fatores (número de sementes e período de embebição), estabeleceu relação direta com o aumento do valor da condutividade na solução de embebição. Essa constatação foi identificada nos diferentes procedimentos estudados. $\mathrm{O}$ valor da condutividade, no menor volume de água, foi superior aos tratamentos com maior volume, indicando maior concentração de lixiviados no menor volume de água de embebição. Com 1 hora de embebição, 
todos os tratamentos aumentaram a quantidade de eletrólitos liberados das sementes, permitindo a classificação dos lotes quanto ao potencial fisiológico por meio da leitura de condutividade elétrica.

Sabendo-se que a liberação inicial de eletrólitos é intensa, tanto pelas sementes intactas e vigorosas como pelas danificadas, torna-se difícil à identificação de possíveis diferenças de qualidade entre os lotes logo no início da embebição. No entanto, com o decorrer deste processo, a quantidade de exsudatos liberados pelas sementes vigorosas vai se estabilizando, em razão, principalmente, da reorganização das membranas, favorecendo a ordenação dos lotes em níveis de qualidade. Sá (1999) observou, em sementes de tomate, que $75 \%$ do total de lixiviados obtidos ocorreram nas seis primeiras horas de embebição. Sendo assim, sementes mais deterioradas ou danificadas liberam maiores quantidades de solutos durante a embebição refletindo o grau de desorganização de suas membranas e, conseqüentemente, sua qualidade fisiológica e vigor.

Examinando os resultados da condutividade elétrica em função do número de sementes, verifica-se que o número de sementes não proporcionou grandes diferenças entre os valores de condutividade elétrica. Sá (1999) também não verificou diferenças significativas entre os valores de condutividade elétrica em função do número de sementes utilizadas na condução dos testes em sementes de tomate. Alguns trabalhos, visando adequar o teste a determinadas espécies, avaliaram diferentes números de sementes, mostrando que é possível reduzir a quantidade de sementes utilizadas e obter uma melhor estratificação dos lotes de sementes (Gaspar e Nakagawa, 2002). Portanto, seria mais indicado utilizar 50 sementes por repetição, devido à economia no tempo e no material empregado para o teste.

Com relação ao período de imersão de sementes de azevém, verificou-se o aumento na quantidade de eletrólitos liberados pelas sementes com o decorrer do período de imersão, ratificando a influência do período de imersão das sementes na quantidade de solutos lixiviados para a solução conforme relatado por Menezes et al., (2007) em sementes de aveia preta, Fessel et al. (2005), em sementes de brócolis e Roveri-José et al., (2001) em sementes de pimentão.

Com o decorrer do período de embebição, a quantidade de exsudatos liberados pelas sementes vigorosas vai se estabilizando, em razão, principalmente, da reorganização das membranas, favorecendo a ordenação dos lotes em níveis de qualidade. Verificou-se que, no período de 1 hora de imersão, já foi possível separar o lote 1 dos demais lotes mostrando que este lote mesmo num curto período de imersão apresentou maior potencial fisiológico. Estes resultados revelam que o teste de condutividade pode ser mais rápido que o usualmente recomendado (24 horas) já que com uma hora foi possível se obter uma indicação do lote de maior vigor (lote 1). Este fato é de grande importância para os programas de controle de qualidade de sementes onde se buscam informações sobre o vigor dos lotes em período de tempo relativamente curto.

A classificação dos lotes foi mais evidente nas combinações proporcionais (número de sementes: volume de água), em que o número de sementes se encontra aproximadamente de 1:1 com o volume de água (50 sementes/ $50 \mathrm{~mL}$ e 100 sementes/ $75 \mathrm{~mL}$ ) (Tabelas 4 e 7). Nestas combinações constatou-se leituras de condutividade elétrica mais próxima dos resultados dos testes prévios de caracterização dos lotes. Usando a combinação 50 sementes e $50 \mathrm{~mL}$ de água (Tabela 4), verificou-se que independente do tempo de embebição, a tendência de classificação dos lotes se manteve. Para espécies de sementes pequenas, o período de embebição pode variar de minutos a poucas horas, como observado para várias espécies (Roveri-José et al., 2001; Gaspar e Nakagawa, 2002).

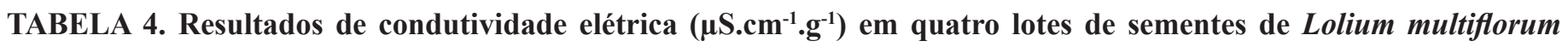
L. de diferentes níveis de qualidade fisiológica em função do número de sementes e volume de água (50 sementes/ $50 \mathrm{~mL}$ ).

\begin{tabular}{|c|c|c|c|c|c|c|}
\hline \multirow{3}{*}{ Lotes } & \multicolumn{6}{|c|}{ Período de embebição (h) } \\
\hline & 1 & 2 & 4 & 6 & 8 & 24 \\
\hline & \multicolumn{6}{|c|}{50 sementes $/ 50 m L$} \\
\hline L1 & 84,6 b & $103,9 \mathrm{~b}$ & $130,4 \mathrm{~b}$ & $144,2 \mathrm{~b}$ & $158,8 \mathrm{~b}$ & $199,2 \mathrm{~b}$ \\
\hline L2 & 157,2 a & 185,9 a & 219,6 a & 239,5 a & $184,6 \mathrm{~b}$ & 306,8 a \\
\hline L3 & 141,6 a & 172,6 a & 213,3 a & 242,0 a & 260,5 a & 311,1 a \\
\hline L4 & 180,1 a & 211,3 a & 257,4 a & 293,1 a & 313,5 a & $400,1 \quad \mathrm{a}$ \\
\hline C.V. & 17,2 & 17,1 & 15,4 & 15,0 & 13,5 & 16,5 \\
\hline
\end{tabular}

Os valores seguidos pela mesma letra, na coluna, não diferem entre si pelo teste de Tukey $(\mathrm{P} \leq 0,05)$. 
TABELA 5. Resultados de condutividade elétrica $\left(\mu \mathrm{S} . \mathrm{cm}^{-1} \cdot \mathrm{g}^{-1}\right)$ em quatro lotes de sementes de Lolium multiflorum $\mathrm{L}$. de diferentes níveis de qualidade fisiológica em função do número de sementes e volume de água (100 sementes/ $50 \mathrm{~mL}$ ).

\begin{tabular}{cccccccc}
\hline & \multicolumn{7}{c}{ Período de embebição (h) } \\
\cline { 2 - 8 } Lotes & 1 & 2 & 4 & 6 & 8 & 24 \\
& \multicolumn{10}{c}{100 sementes/50mL } \\
\hline L1 & $85,1 \mathrm{~b}$ & $102,4 \mathrm{~b}$ & $128,5 \mathrm{~b}$ & $145,8 \mathrm{~b}$ & $158,9 \mathrm{~b}$ & $188,3 \mathrm{~b}$ \\
L2 & $129,1 \mathrm{ab}$ & $150,0 \mathrm{ab}$ & $181,5 \mathrm{ab}$ & $201,5 \mathrm{ab}$ & 214,6 ab & $254,5 \mathrm{ab}$ \\
L3 & $150,9 \mathrm{a}$ & $173,2 \mathrm{ab}$ & $227,1 \mathrm{a}$ & $250,0 \mathrm{a}$ & $267,5 \mathrm{a}$ & $305,5 \mathrm{a}$ \\
L4 & $162,3 \mathrm{a}$ & $193,1 \mathrm{a}$ & $245,6 \mathrm{a}$ & $267,3 \mathrm{a}$ & $281,1 \mathrm{a}$ & $326,6 \mathrm{a}$ \\
\hline C.V. & 21,8 & 21,9 & 20,0 & 20,1 & 20,1 & 19,4 \\
\hline
\end{tabular}

Os valores seguidos pela mesma letra, na coluna, não diferem entre si pelo teste de Tukey $(\mathrm{P} \leq 0,05)$.

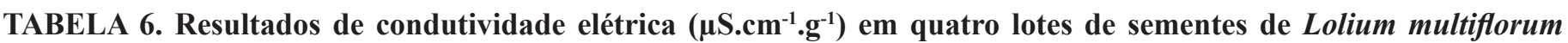
L. de diferentes níveis de qualidade fisiológica em função do número de sementes e volume de água (50 sementes/ $75 \mathrm{~mL}$ ).

\begin{tabular}{|c|c|c|c|c|c|c|}
\hline \multirow{3}{*}{ Lotes } & \multicolumn{6}{|c|}{ Período de embebição (h) } \\
\hline & 1 & 2 & 4 & 6 & 8 & 24 \\
\hline & \multicolumn{6}{|c|}{50 sementes $/ 75 \mathrm{~mL}$} \\
\hline L1 & 73,9 b & 88,7 b & $112,6 \mathrm{~b}$ & $129,5 \mathrm{~b}$ & $140,2 \mathrm{~b}$ & 192,4 c \\
\hline L2 & $94,8 \mathrm{ab}$ & $116,8 \mathrm{ab}$ & $143,5 \mathrm{ab}$ & $163,6 \mathrm{ab}$ & $175,5 \mathrm{ab}$ & 226,7 c \\
\hline L3 & 111,6 a & 129,6 a & 156,2 a & 173,9 a & 184,6 a & 243,1 c \\
\hline L4 & 109,3 a & 127,1 a & 159,8 a & $178,7 \mathrm{a}$ & $192,5 \mathrm{a}$ & 248,9 c \\
\hline C.V. & 13,9 & 13,6 & 11,6 & 11,8 & 10,7 & 13,7 \\
\hline
\end{tabular}

Os valores seguidos pela mesma letra, na coluna, não diferem entre si pelo teste de Tukey $(\mathrm{P} \leq 0,05)$.

TABELA 7. Resultados de condutividade elétrica $\left(\mu \mathrm{S}_{\mathrm{C}} \mathrm{cm}^{-1} \cdot \mathrm{g}^{-1}\right)$ em quatro lotes de sementes de Lolium multiflorum $\mathrm{L}$. de diferentes níveis de qualidade fisiológica em função do número de sementes e volume de água (100 sementes/ $75 \mathrm{~mL}$ ).

\begin{tabular}{ccccccc}
\hline & \multicolumn{5}{c}{ Período de embebição (h) } \\
\cline { 2 - 6 } Lotes & 1 & 2 & 4 & 6 & 8 & 24 \\
\cline { 2 - 6 } & \multicolumn{7}{c}{100 sementes $/ 75 m L$} \\
\hline L1 & $60,4 \mathrm{~b}$ & $76,2 \mathrm{~b}$ & $91,2 \mathrm{~b}$ & $100,3 \mathrm{~b}$ & $108,3 \mathrm{~b}$ & $129,6 \mathrm{~b}$ \\
L2 & $91,2 \mathrm{a}$ & $113,3 \mathrm{a}$ & $126,4 \mathrm{a}$ & $140,0 \mathrm{a}$ & $149,0 \mathrm{a}$ & $169,0 \mathrm{ab}$ \\
L3 & $99,7 \mathrm{a}$ & $129,9 \mathrm{a}$ & $155,0 \mathrm{a}$ & $167,8 \mathrm{a}$ & $178,2 \mathrm{a}$ & $199,2 \mathrm{a}$ \\
L4 & $97,0 \mathrm{a}$ & $119,1 \mathrm{a}$ & $145,5 \mathrm{a}$ & $159,8 \mathrm{a}$ & $170,4 \mathrm{a}$ & $202,1 \mathrm{a}$ \\
\hline C.V. & 12,1 & 12,0 & 12,3 & 12,3 & 11,1 & 12,4 \\
\hline
\end{tabular}

Os valores seguidos pela mesma letra, na coluna, não diferem entre si pelo teste de Tukey $(\mathrm{P} \leq 0,05)$.

Considera-se que os objetivos básicos dos testes de vigor são: a identificação de diferenças no potencial fisiológico de lotes com germinação semelhante, distinção, com segurança, lotes de alto e baixo vigor, de maneira comparável à emergência de plântulas em campo (Marcos Filho, 1999). De modo geral, a mensuração dos exsudatos liberados pelas sementes mediante a condutividade elétrica na separação dos níveis de qualidade fisiológica de sementes de azevém 
foi eficiente, pois os resultados foram semelhantes aos dos testes convencionais. Considerando as características desejáveis para adoção de um teste de vigor como rotina num laboratório de análise de sementes, o teste de condutividade elétrica parece ser o mais indicado devido sua facilidade na execução, objetividade e rapidez.

\section{CONCLUSÕES}

O teste de condutividade elétrica conduzido com quatro sub-amostras de 50 sementes puras embebidas em $50 \mathrm{~mL}$ de água deionizada, na temperatura de $25{ }^{\circ} \mathrm{C}$, permitiu a separação dos lotes a partir de 1 hora de embebição, mostrando-se promissor na avaliação da qualidade das sementes de azevém.

\section{REFERÊNCIAS}

BRASIL. Ministério da Agricultura e Reforma Agrária. Secretaria Nacional de Defesa Agropecuária. Departamento Nacional de Produção Vegetal. Coordenação de Laboratório Vegetal. Regras para análise de sementes. Brasília, DF, 1992. 365p.

BRESOLIN, A.P.S. Avaliação de populações de azevém quanto à tolerância ao alumínio tóxico e estimativa de tamanho de amostra para estudos de diversidade genética com marcadores AFLP. 2007. 76f. Dissertação (Mestrado em Agronomia) - Faculdade de Agronomia Eliseu Maciel, Universidade Federal de Pelotas, Pelotas, 2007.

CARVAlho, N.M.; NAKAGAWA, J. Sementes: ciência, tecnologia e produção. 4ed. Jaboticabal: FUNEP, 2000. 424p.

DIAS, D.C.F.S. BHERING, M.C.; TOKUHISA, D.; HILST, P.C. Teste de condutividade elétrica para avaliação do vigor em sementes de cebola. Revista Brasileira de Sementes, v.28, n.1, p.154-162, 2006.

DIAS, D.C.F.S.; MARCOS-FILHO, J. Testes de condutividade elétrica para avaliação do vigor de sementes de soja (Glycine $\max$ (L.) Merrill). Scientia Agricola, v.53, n.1, p.31-42, 1996.

FESSEL, S.A.; SILVA, L.J.R.; SADER, R. Teste de condutividade elétrica para avaliar a qualidade fisiológica de sementes de brócolis (Brassica oleracea L. var. italica Plenk). Científica, v.33, n.1, p.35-41, 2005.

GARCIA, D.C.; MENEZES, N.L. Teste de envelhecimento acelerado para sementes de azevém, aveia preta e milheto. Ciência Rural, v.29, n.2, p. 233-237, 1999.
GASPAR, C.M.; NAKAGAWA, J. Teste de condutividade elétrica em função do número de sementes e da quantidade de água para sementes de milheto. Revista Brasileira de Sementes, v.24, n.2, p.70-76, 2002.

HAMPTON, J.G.; COOLBEAR, P. Potential versus actual seed performance - can vigour testing provide an answer? Seed Science and Technology, v.18, n.2, p.215-228, 1990.

MARCOS FILHO, J. Fisiologia de sementes de plantas cultivadas. Piracicaba: FEALQ, 2005. 495p.

MARCOS FILHO, J. Teste de envelhecimento acelerado. In: KRZYZANOWSKI, F.C.; VIEIRA, R.D.; FRANÇA NETO, J.B. (Eds.). Vigor de sementes: conceitos e testes. Londrina: ABRATES, 1999. cap.3, p.1-24.

MENEZES, N.L.; GARCIA, D.C.; BAHRY, C.A.; MATTIONI, N.M. Teste de condutividade elétrica em aveia preta. Revista Brasileira de Sementes, v.29, n.2, p.138142, 2007.

PEREIRA, A.V.; MITTELMANN, A.; LEDO, F.J.S.; SOUZA SOBRINHO, F.; AUAD, A.M.; OLIVEIRA, J.S. Comportamento agronômico de azevém anual (Lolium multiflorum L.) para cultivo invernal na região sudeste. Ciência e Agrotecnologia, v.32, n.2, p.567-572, 2008.

POPINIGIS, F. Fisiologia da semente. Brasília, DF: Associação Brasileira de Educação Agrícola Superior/ Ministério da Educação e Cultura (ABEAS/MEC), 1985. 289p.

ROVERI-JOSÉ, S.C.B.; CARVALHO, M.L.M. de; RODRIGUES, R. Teste de condutividade elétrica para avaliação da qualidade fisiológica de sementes de pimentão (Capsicum annuum L.). Revista Brasileira de Sementes, v.23, n.1, p.55-61, 2001.

SÁ, M.E. Condutividade elétrica em sementes de tomate (Lycopersicon lycopersicum L.). Scientia Agrícola, v.56, n.1, p.13-19, 1999.

SANTOS, H.P.; FONTANELI, R.S.; BAIER, A.C. TOMM, G.O. Principais forrageiras para integração lavourapecuária, sob plantio direto, nas regiões planalto e Missões do Rio Grande do Sul. Passo Fundo: Embrapa Trigo, 2002. 142p.

STATISTICAL ANALYSIS SYSTEM - SAS. SAS OnlineDoc. Version 9.1.3. Cary: SAS Institute, 2004. (CDROM).

TERRA-LOPES, M.L.; CARVALHO, P.C.F.; ANGHINONI, I.; SANTOS, D.T.; AGUINAGA, A.A.Q.; FLORES, J.P.C.; MORAES, A. Sistema de integração lavoura-pecuária: efeito do manejo da altura em pastagem de aveia 
preta e azevém anual sobre o rendimento da cultura da soja. Ciência Rural, v.39, n.5, p.1499-1506, 2009.

VIEIRA, R.D.; KRZYZANOWSKI, F.C. Teste de condutividade elétrica. In: KRZYZANOWSKI, F.C.; VIEIRA, R.D.; FRANÇA NETO, J.B. (Ed.). Vigor de sementes: conceitos e testes. Londrina: ABRATES, 1999. cap.4, p.1-26.
VIEIRA, R.D.; PENARIOL, A.L.; PERECIN, D.; PANOBIANO, M. Condutividade elétrica e o teor de água inicial das sementes de soja. Pesquisa Agropecuária Brasileira, v.37, n.19, p.1333-1338, 2002. 